\title{
Gravitational anomaly and Hawking radiation of apparent horizon in FRW universe
}

\author{
Ran Li ${ }^{\mathrm{a}}$, Ji-Rong Ren ${ }^{\mathrm{b}}$, Shao-Wen Wei \\ Institute of Theoretical Physics, Lanzhou University, Lanzhou 730000, Gansu, China
}

Received: 27 February 2009 / Published online: 26 June 2009

(C) Springer-Verlag / Società Italiana di Fisica 2009

\begin{abstract}
Motivated by the successful applications of the anomaly cancellation method to derive Hawking radiation from various types of black hole spacetimes, we further extend the gravitational anomaly method to investigate the Hawking radiation from the apparent horizon of a FRW universe by assuming that the gravitational anomaly also exists near the apparent horizon of the FRW universe. The result shows that the radiation flux from the apparent horizon of the FRW universe measured by a Kodama observer is just the pure thermal flux. The result presented here will further confirm the thermal properties of the apparent horizon in a FRW universe.
\end{abstract}

PACS $04.62 .+v \cdot 04.70 . D y \cdot 11.30-\mathrm{j}$

Using the techniques of quantum field theory in a curved spacetime background, Hawking [1, 2] firstly discovered the thermal radiation of a collapsing black hole. Since the Hawking radiation relates the theory of general relativity with quantum field theory and statistical thermodynamics, it is generally believed that a deeper understanding of Hawking radiation may shed some light on seeking the underlying quantum gravity. Due to the difficulties existing in extending the Hawking's original method to more complicated spacetime backgrounds, several derivations of Hawking radiation have been proposed in the literature, including the so-called Damour-Ruffini method [3], the trace anomaly method [4], the quantum tunneling method [5] and the gravitational anomaly method $[6,7]$.

A recent proposal of deriving Hawking radiation via gravitational and gauge anomalies proposed by Wilczek and his collaborators $[6,7]$ has attracted a lot of interest. This rejuvenates the interest of investigation for Hawking radiation. For various types of black holes, these investigations

\footnotetext{
a e-mail: liran05@1zu.cn

b e-mail: renjr@1zu.edu.cn
}

have been carried out [8-28]. In fact, the anomaly analysis can be traced back to Christensen and Fulling's early work [29], in which they suggested that there exists a relation between the Hawking radiation and the anomalous trace of the field under the condition that the covariant conservation law is valid. Imposing boundary condition near the horizon, Wilczek et al. showed that Hawking radiation is just the cancel term of the gravitational anomaly of the covariant conservation law and gauge invariance. Their basic idea is that, near the horizon, a quantum field in a black hole background can be effectively described by an infinite collection of $(1+1)$-dimensional fields on $(t, r)$ space, where $r$ is the radial direction. One could treat the original higher dimensional field as a collection of two-dimensional quantum fields using dimensional reduction. This is because, near the horizon, the property of the black hole metric, mass or potential terms for quantum fields in it can be suppressed. In this two-dimensional reduction, because all the ingoing modes cannot classically affect physics outside the horizon, the two-dimensional effective action in the exterior region becomes anomalous with respect to gauge or general coordinate symmetries. To cancel the anomaly, they found that the Hawking flux is universally determined only by the value of anomalies at the horizon.

In the present work, motivated by the successful applications of the anomaly cancellation method to derive Hawking radiation from various types of black hole spacetimes, we further extend the gravitational anomaly method to investigate the Hawking radiation from apparent horizon of FRW universe by assuming that the gravitational anomaly also exists near the apparent horizon of a FRW universe. The hypothesis that a gravitational anomaly exists near the apparent horizon of a FRW universe is reasonable because the apparent horizon of a FRW universe can be viewed as a causal boundary and previous investigations show that the apparent horizon is thermal like the event horizon of a black hole. Under this hypothesis one can derive the Hawking radiation from the apparent horizon of a FRW universe us- 
ing the general gravitational anomaly formalism. The result shows that the radiation flux from the apparent horizon of FRW universe measured by a Kodama observer is just the pure thermal flux. The result presented here will further confirm the thermal properties of the apparent horizon of the FRW universe.

Recently, the thermal properties of the apparent horizon of the FRW universe have attracted a lot of interest. In fact, the investigations can be traced back to Jacobson's work [30], in which the Einstein equation can be derived from the proportionality of entropy and horizon area together with the fundamental Clausius relation $\delta Q=T d S$ connecting heat, entropy, and temperature. It has been proved that the above idea can also be applied in establishing the relationship between the Friedmann equations and the first law of thermodynamics in the framework of the Friedmann-RobertsonWalker (FRW) universe. Assuming that the apparent horizon of a FRW universe has temperature $T=1 / 2 \pi \tilde{r}_{A}$ and entropy $S=A / 4$, where $\tilde{r}_{A}$ and $A$ are the radius and the area of the apparent horizon, respectively, the Friedmann equations can be derived from the Clausius relation [32] and the first Friedmann equation can be cast into the form of the unified first law [31,33]. For some related investigations see also [34-42].

However, whether there is a Hawking temperature associated with the apparent horizon of a FRW universe is still an assumption. In a recent paper [43], the scalar particles' Hawking radiation from the apparent horizon of FRW universe was investigated by using the tunneling formalism. There it was found that the Kodama observer inside the apparent horizon does see a thermal spectrum with temperature $T=1 / 2 \pi \tilde{r}_{A}$, which is caused by particles tunneling from outside the apparent horizon to inside the apparent horizon. Subsequently, we considered the fermions' tunneling from the apparent horizon in [44], where the radiation spectrum and Hawking temperature are correctly recovered. The results in the present paper together with that in [43] and [44] will fill in the gap existing in the literature investigating the relationship between the first law of thermodynamics and Friedmann equations and confirm the thermal properties of the apparent horizon.

For convenience, we firstly review some results related to the FRW universe. The FRW universe is described by the metric

$d s^{2}=-d t^{2}+a^{2}(t)\left(\frac{d r^{2}}{1-k r^{2}}+r^{2}\left(d \theta^{2}+\sin ^{2} \theta d \phi^{2}\right)\right)$,

where $a(t)$ is the scale factor and $k=1,0$ and -1 represent the closed, flat and open universe, respectively. Introducing $R=a r$, the FRW metric (1) can be rewritten as

$d s^{2}=h_{a b} d x^{a} d x^{b}+R^{2} d \Omega_{2}^{2}$, where $x^{a}=(t, r), h_{a b}=\operatorname{diag}\left(-1, \frac{a^{2}}{1-k r^{2}}\right)$ and $d \Omega_{2}^{2}$ represents the line element of $S^{2}$. The apparent horizon is defined by the equation

$h^{a b} \partial_{a} R \partial_{b} R=0$,

which gives us the location of apparent horizon explicitly as

$R_{A}=\frac{1}{\sqrt{H^{2}+k / a^{2}}}$,

with $H=\dot{a} / a$ being the Hubble parameter. The temperature of the apparent horizon is defined by $T=\frac{|\kappa|}{2 \pi}$, where the surface gravity $\kappa$ is given by

$\kappa=\left.\frac{1}{2 \sqrt{-h}} \partial_{a}\left(\sqrt{-h} h^{a b} \partial_{b} R\right)\right|_{R=R_{A}}=-\frac{1}{R_{A}}(1-\epsilon)$,

with $\epsilon=\frac{\dot{R}_{A}}{2 H R_{A}}$. In this paper, we will adopt the approximation $\epsilon \ll 1$ to simplify the calculation in the following. This approximation is also supported by the investigations about the Hawking radiation from the apparent horizon in the tunneling approach. Under the approximation, the temperature of apparent horizon is simply given by $T=\frac{1}{2 \pi R_{A}}$.

For simplicity, we will use the $(t, R)$ coordinates, in which the FRW metric can be rewritten as

$$
\begin{aligned}
d s^{2}= & -\frac{1-R^{2} / R_{A}^{2}}{1-k R^{2} / a^{2}} d t^{2}-\frac{2 H R}{1-k R^{2} / a^{2}} d t d R \\
& +\frac{1}{1-k R^{2} / a^{2}} d R^{2}+R^{2} d \Omega_{2}^{2} .
\end{aligned}
$$

One can diagonalize the metric by introducing the coordinates transformation

$d t^{\prime}=d t+\frac{H R}{1-R^{2} / R_{A}^{2}} d R$.

The FRW metric in the new coordinates is of the form

$d s^{2}=-\frac{1-R^{2} / R_{A}^{2}}{1-k R^{2} / a^{2}} d t^{2}+\frac{1}{1-R^{2} / R_{A}^{2}} d R^{2}+R^{2} d \Omega_{2}^{2}$.

In this paper, we want to investigate the thermal flux measured by a Kodama observer. So we must introduce the time $T$ measured by a Kodama observer. The relationship between Kodama time $T$ and time $t$ is given by

$d T=\frac{1}{\sqrt{1-k R^{2} / a^{2}}} d t$.

Finally, the FRW metric reads

$$
\begin{aligned}
d s^{2}= & -\left(1-R^{2} / R_{A}^{2}\right) d T^{2} \\
& +\frac{1}{1-R^{2} / R_{A}^{2}} d R^{2}+R^{2} d \Omega_{2}^{2} .
\end{aligned}
$$


Therefore, after dimensional reduction, the physics near the apparent horizon can be effectively described by the $(1+1)$ dimensional metric

$d s^{2}=-\left(1-R^{2} / R_{A}^{2}\right) d T^{2}+\frac{1}{1-R^{2} / R_{A}^{2}} d R^{2}$.

Now, by assuming that the gravitational anomaly also exists near the apparent horizon of a FRW universe, we will apply the anomaly method to calculate the radiation flux from the apparent horizon. It should be noted that the approximation $\epsilon \ll 1$ is implied in the following calculations. So we can just treat the related physical quantities as independent of the Kodama time $T$. In $(1+1)$ dimensions, the anomaly for the energy-momentum tensor is given by

$\nabla_{\mu} T_{\nu}^{\mu}=\frac{1}{\sqrt{-g}} \partial_{\mu} N_{v}^{\mu}=\frac{1}{96 \pi \sqrt{-g}} \epsilon^{\beta \delta} \partial_{\delta} \partial_{\alpha} \Gamma_{\nu \beta}^{\alpha}$.

The energy-momentum tensor can be written as

$T_{v}^{\mu}=T_{v(i)}^{\mu} \Theta_{-}(R)+T_{v(H)}^{\mu} H(R)$,

where $\Theta_{-}(R)=\Theta\left(R_{A}-R+\epsilon\right)$ and $H(R)=1-\Theta_{-}(R)$. Inside the apparent horizon, we have

$\partial_{R} T_{T(i)}^{R}=0$.

Near the horizon, we have the anomalous equation

$\partial_{R} T_{T(H)}^{R}=\partial_{R} N_{T}^{R}$.

These two equations can also be integrated as

$T_{T(i)}^{R}=a_{i}$

$T_{T(H)}^{R}=a_{H}+\int_{R_{A}}^{R} d R \partial_{R} N_{T}^{R}$.

Under the infinitesimal general coordinate transformations, the effective action changes as

$$
\begin{aligned}
-\delta W= & \int d^{2} x \xi^{T} \nabla_{\mu} T_{T}^{\mu} \\
= & \int d^{2} x \xi^{T}\left[\partial_{R}\left(N_{T}^{R} H\right)\right. \\
& \left.-\delta\left(R_{A}-R+\epsilon\right)\left(T_{T(i)}^{R}-T_{T(H)}^{R}+N_{T}^{R}\right)\right] .
\end{aligned}
$$

The coefficient of the delta function term should also vanish at the horizon

$a_{i}=a_{H}-N_{T}^{R}\left(R_{A}\right)$.

We impose a vanishing condition for the covariant energymomentum tensor at the horizon, which gives the equation

$a_{H}=2 N_{T}^{R}\left(R_{A}\right)$.
The total flux of the energy-momentum tensor measured by the Kodama observer inside the apparent horizon is given by

$a_{i}=N_{T}^{R}\left(R_{A}\right)$

From the effective two-dimensional metric (11), $N_{T}^{R}$ is given by

$N_{T}^{R}\left(R_{A}\right)=\frac{1}{12} \pi T^{2}$

where $T=\frac{1}{2 \pi R_{A}}$ is the temperature of apparent horizon. This result indicates that the Kodama observer inside the apparent horizon does see a thermal spectrum with the temperature $T=\frac{1}{2 \pi R_{A}}$.

In summary, we have extended the gravitational anomaly method to investigate the Hawking radiation from the apparent horizon of a FRW universe by assuming that a gravitational anomaly also exists near the apparent horizon of a FRW universe. The result shows that the radiation flux from the apparent horizon of a FRW universe measured by a Kodama observer is just the pure thermal flux. Although the gravitational anomaly of the apparent horizon is just an assumption in the present paper, the final result is consistent with that in other approaches, which supports the thermal properties of apparent horizon. The results in the present paper together with those in [43] and [44] will fill in the gap existing in the literature investigating the relationship between the first law of thermodynamics and Friedmann equations.

Acknowledgements This work was supported by the National Natural Science Foundation of China and Cuiying Project of Lanzhou University.

\section{References}

1. S.W. Hawking, Nature 248, 30 (1974)

2. S.W. Hawking, Commun. Math. Phys. 43, 199 (1975)

3. T. Damour, R. Ruffini, Phys. Rev. D 14, 332 (1976)

4. S. Christensen, S. Fulling, Phys. Rev. D 15, 2088 (1977)

5. M.K. Parikh, F. Wilczek, Phys. Rev. Lett. 85, 5042 (2000)

6. S.P. Robinson, F. Wilczek, Phys. Rev. Lett. 95, 011303 (2005)

7. S. Iso, H. Umetsu, F. Wilczek, Phys. Rev. Lett. 96, 151302 (2006)

8. S. Iso, H. Umetsu, F. Wilczek, Phys. Rev. D 74, 044017 (2006)

9. K. Murata, J. Soda, Phys. Rev. D 74, 044018 (2006)

10. M.R. Setare, Eur. Phys. J. C 49, 865 (2007)

11. S. Iso, T. Morita, H. Umetsu, J. High Energy Phys. 04, 068 (2007)

12. Q.-Q. Jiang, S.-Q. Wu, Phys. Lett. B 647, 200 (2007)

13. Q.-Q. Jiang, S.-Q. Wu, X. Cai, Phys. Rev. D 75, 064029 (2007)

14. K. Xiao, W. Liu, H. Zhang, Phys. Lett. B 647, 482 (2007)

15. H. Shin, W. Kim, J. High Energy Phys. 06, 012 (2007)

16. Q.-Q. Jiang, S.-Q. Wu, X. Cai, Phys. Lett. B 651, 65 (2007)

17. S.-Q. Wu, J.-J. Peng, Class. Quantum Gravity 24, 5123 (2007)

18. W. Kim, H. Shin, J. High Energy Phys. 07, 070 (2007)

19. K. Murata, U. Miyamoto, Phys. Rev. D 76, 084038 (2007)

20. R. Banerjee, S. Kulkarni, Phys. Rev. D 77, 024018 (2008)

21. S. Gangopadhyay, S. Kulkarni, Phys. Rev. D 77, 024038 (2008)

22. J.-J. Peng, S.-Q. Wu, Phys. Lett. B 661, 300 (2008)

23. X. Wu, C.-G. Huang, J.-R. Sun, Phys. Rev. D 77, 124023 (2008) 
24. S.-Q. Wu, J.-J. Peng, Z.-Y. Zhao, Class. Quantum Gravity 25, 135001 (2008)

25. S. Gangopadhyay, Phys. Rev. D 78, 044026 (2008)

26. E. Papantonopoulos, P. Skamagoulis, arXiv:0812.1759

27. S.-W. Wei, R. Li, Y.-X. Liu, J.-R. Ren, arXiv:0901.2614

28. S. Nam, J.-D. Park, arXiv:0902.0982

29. S.M. Christensen, S.A. Fulling, Phys. Rev. D 15, 2088 (1977)

30. T. Jacobson, Phys. Rev. Lett. 75, 1260 (1995)

31. R.G. Cai, L.M. Cao, Phys. Rev. D 75, 064008 (2007)

32. R.G. Cai, S.P. Kim, J. High Energy Phys. 0502, 050 (2005)

33. M. Akbar, R.G. Cai, Phys. Rev. D 75, 084003 (2007)

34. M. Akbar, R.G. Cai, Phys. Lett. B 635, 7 (2006)
35. M. Akbar, R.G. Cai, Phys. Lett. B 648, 243 (2007)

36. R.G. Cai, L.M. Cao, Nucl. Phys. B 779, 1 (2007)

37. R.G. Cai, L.M. Cao, Y.P. Hu, J. High Energy Phys. 0808, 090 (2008)

38. X.H. Ge, Phys. Lett. B 651, 49 (2007)

39. Y.G. Gong, A.Z. Wang, Phys. Rev. Lett. 99, 211301 (2007)

40. S.F. Wu, B. Wang, G.H. Yang, Nucl. Phys. B 799, 330 (2008)

41. T. Zhu, J.R. Ren, S.F. Mo, arXiv:0805.1162

42. M. Akbar, arXiv:0808.0169

43. R.-G. Cai, L.-M. Cao, Y.-P. Hu, arXiv:0809.1554

44. R. Li, J.R. Ren, D.F. Shi, Phys. Lett. B 670, 446 (2009) 